\title{
ON POLYNOMIAL SOLUTIONS OF A CLASS OF LINEAR DIFFERENTIAL EQUATIONS OF THE SECOND ORDER*
}

\author{
BY W. C. BRENKE
}

1. Introduction. Certain well known polynomials have a number of common properties. They arise as coefficients of $t^{n}$ in the expansion of a generating function; they may be obtained by means of orthogonalization of a set of functions $x^{n} g(x)$ when the function $\rho(x)=g^{2}(x)$ and the interval are properly chosen; they may be regarded as polynomials which become orthogonal when multiplied by a proper factor $g(x)$; they satisfy a certain type of difference equation; they satisfy a certain type of differential equation. The results of this paper are based on the differential equation. Some of them are general statements of results already known for various classes of polynomials; others are believed to be new.

2. Polynomial Solutions. Consider the differential equation

$$
P(x) y_{n}^{\prime \prime}+Q(x) y_{n}^{\prime}+\lambda_{n} R(x) y_{n}=0,
$$

where the coefficients $P, Q, R$ are assumed to be real polynomials in the real variable $x$ none of which vanish identically and $\lambda_{n}$ is a polynomial in $n$. We assume that $y_{n}$ is a polynomial of the form $\dagger$

$$
\begin{aligned}
y_{n}=x^{n}+C_{1}{ }^{(n)} x^{n-1}+C_{2}(n) x^{n-2}+\cdots+ & C_{n}^{(n)}, \\
& (n=0,1,2, \cdots),
\end{aligned}
$$

where the coefficient of the highest power of $x$ is unity. On substituting $y_{0}=1, y_{1}=x+a_{1}, y_{2}=x^{2}+a x+b$, we find that $P$ and $Q$ must contain the factor $R$. Dividing out this factor and putting $\lambda_{1}=1$, as may be done without loss of generality, we find that the differential equation must have the form

* Presented to the Society, November 26, 1927, and December 1, 1928.

$\dagger$ When reference is made to various standard polynomials it will be assumed that they have been put into this form by use of a suitable factor. 


$$
p y_{n}^{\prime \prime}+q y_{n}^{\prime}+\lambda_{n} y_{n}=0,
$$

where

(4) $p=\alpha x^{2}+\beta x+\gamma, q=-\left(x+a_{1}\right), \lambda_{n}=n-n(n-1) \alpha$,

the value of $\lambda_{n}$ being uniquely determined when $\alpha$ is given.

For any assigned values of the constants $\alpha, \beta, \gamma, a_{1}$, the equation (3) will then give a complete system of polynomials starting with $y_{0}=1$ and $y_{1}=x+a_{1}$, provided that the parameter values $\lambda_{n}$ are all distinct. The values of the coefficients in (2) will then be

$$
\begin{aligned}
C_{1}{ }^{(n)}= & \frac{n a_{1}-n(n-1) \beta}{\lambda_{n}-\lambda_{n-1}} ; \\
C_{t}{ }^{(n)}= & \frac{(n-t+1)\left[a_{1}-(n-t) \beta\right] C_{t-1}^{(n)}}{\lambda_{\iota}-\lambda_{n-t}} \\
& -\frac{(n-t+2)(n-t+1) C_{t-2}^{(n)}}{\lambda_{n}-\lambda_{n-t}} ; \quad(t=2,3, \cdots n) ; \\
C_{0}{ }^{(n)}= & 0 .
\end{aligned}
$$

The case of equal parameter values will be considered under $\S 9$.

3. Orthogonal Systems. These polynomials will form an orthogonal system if the equation $p=0$ has real and distinct roots $x=a$ and $x=b$, infinite roots not excluded, and if the constants $\alpha, \beta, \gamma, a_{1}$ are conditioned by certain inequalities. We shall assume $a<b$. To obtain these inequalities we first find, by the usual process, that

provided that

$$
\int_{a}^{b} \rho y_{n} y_{m} d x=0, \quad n \neq m,
$$

$$
\rho p=e^{\mathcal{S}(q / p) d x}, \text { and } \rho p=0 \text { at } a \text { and } b .
$$

Then, if we assume that $\rho(x)$ is not negative in $(a, b)$, the functions $\rho^{1 / 2} y_{n}$ form an orthogonal system for the interval $(a, b)$, which may reach to infinity in either or both directions. This leads to the consideration of the following three cases.

CASE 1.

$$
\begin{aligned}
& \alpha \neq 0 ; \quad p=-\alpha(x-a)(b-x) ; \\
& a, b \text { real and finite; } \quad a<b .
\end{aligned}
$$


The value of $\rho$ from (5) will be

$$
\rho=-\frac{1}{\alpha}(x-a)^{A-1}(b-x)^{B-1},
$$

where

$$
A=\frac{a+a_{1}}{\alpha(b-a)}, \quad B=-\frac{b+a_{1}}{\alpha(b-a)} .
$$

The existence of $\int_{a}^{b} \rho d x$ will require the inequalities $A>0$, $B>0$, that is,

$$
\begin{aligned}
& a+a_{1}>0 \text { and } b+a_{1}<0 \text { if } \alpha>0 ; \\
& a+a_{1}<0 \text { and } b+a_{1}>0 \text { if } \alpha<0 .
\end{aligned}
$$

The case $\alpha>0$ leads to a contradiction since $a<b$; the other is equivalent to

$$
\alpha<0 ;-b<a_{1}<-a .
$$

Then also $\rho p=0$ at $a$ and $b$, and $p$ and $\rho$ are positive in $(a, b)$. This gives the Jacobi polynomials.

Case 2.

$$
\begin{gathered}
\alpha=0 ; \beta \neq 0 ; p=\beta\left(x+\frac{\gamma}{\beta}\right) . \\
\beta<0 \text { gives : } p(x) \geqq 0 \text { in }(a, b)=(-\infty,-\gamma / \beta) ; \\
\beta>0 \text { gives : } p(x) \geqq 0 \text { in }(a, b)=(-\gamma / \beta, \infty) .
\end{gathered}
$$

Equation (5) now gives

$$
\rho= \pm \frac{1}{\beta} e^{-x / \beta}( \pm x \pm \gamma / \beta)^{-\left(a_{1}-\gamma / \beta\right) / \beta-1},
$$

where the upper or the lower signs are to be taken according as $\beta$ is greater than or less than zero.

The existence of $\int_{a}^{b} \rho d x$ requires that $\left(a_{1}-\gamma / \beta\right) / \beta<0$, that is,

$$
a_{1} \lesseqgtr \gamma / \beta \text { according as } \beta \gtrless 0 \text {. }
$$

Then $\rho p$ vanishes at $a$ and $b$, and $\rho$ and $p$ are positive in $(a, b)$. We obtain the generalized polynomials of Laguerre.

Case 3.

$$
\alpha=\beta=0 ; \quad p=\gamma \neq 0 ; \quad(a, b)=(-\infty, \infty) .
$$


Here

$$
\rho=\frac{1}{\gamma} e^{\left.-i x+a_{1}\right)^{2} /(2 \gamma)} .
$$

The existence of $\int_{a}^{b} \rho d x$ requires $\gamma>0$, which also makes $\rho p$ vanish at $a$ and $b$, and $\rho>0$ in $(a, b)$. This gives the general Hermite polynomials.

As examples we may consider the differential equations

$$
(x \pm 1) y_{n}^{\prime \prime}-x y_{n}^{\prime}+n y_{n}=0 .
$$

Polynomial solutions are:

(With the plus sign) (With the minus sign)

$$
\begin{array}{ll}
y_{0}=1, & y_{0}=1, \\
y_{1}=x, & y_{1}=x, \\
y_{2}=x^{2}-2 x-1, & y_{2}=x^{2}-2 x+1, \\
y_{3}=x^{3}-6 x^{2}+3 x+4, & y_{3}=x^{3}-6 x^{2}+9 x-4,
\end{array}
$$

The first set forms an orthogonal system, the second does not.

4. Normalizing Constants. The polynomials obtained in $\$ 3$ form an orthogonal system and hence satisfy a difference equation of the form*

$$
y_{n}=\left(a_{n}+x\right) y_{n-1}-b_{n} y_{n-2}, \quad(n>1) .
$$

Here

$$
\begin{array}{rlrl}
b_{n} & =c_{n-2}^{2} / c_{n-1}^{2}, & a_{n} & =-c_{n-1}^{2} k_{n-1} ; \\
1 / c_{n}^{2} & =\int_{a}^{b} \rho y_{n}^{2} d x, \quad k_{n}=\int_{a}^{b} \rho x y_{n}^{2} d x .
\end{array}
$$

The constants $c_{n}$ are the normalizing constants for the polynomials $y_{n}$, so that, if $f_{n}=\rho^{1 / 2} c_{n} y_{n}$, then

* J. Shohat, Sur le développement de l'intégrale . . et sur les polynomes de Tchebycheff, Rendiconti di Palermo, vol. 47 (1923), pp. 25-46. The case $(a, b)$ finite has also been treated by G. Darboux, Mémoire sur l'approximation des fonctions de très-grands nombres, Journal de Mathématiques, (3), vol. 4 (1878); and by N. Abramescu, Sulle serie di polinomi di una variable complessa. Le serie di Darboux, Annali di Matematica, (3), vol. 31 (1922), pp. 207-249. 


$$
\int_{a}^{b} f_{n} f_{m} d x=1 \text { or } 0
$$

according as $n$ and $m$ are equal or unequal.

Direct substitution of (2) in (6) shows that $a_{n}$ and $b_{n}$ may also be obtained by means of the equations

$$
a_{n}=C_{1}^{(n)}-C_{1}^{(n-1)} ; \quad b_{n}=a_{n} C_{1}^{(n-1)}-C_{2}^{(n)}+C_{2}^{(n-1)} .
$$

When the constants $b_{n}$ are known, the normalizing constants are given by the formula

$$
1 /{c_{n}}^{2}=\left[\prod_{2}^{n+1} b_{r}\right] \int_{a}^{b} \rho d x .
$$

5. Determinant Form of the Difference Equation. The solution of the difference equation (6) may be written in determinant form as

$$
y_{n}=\left|\begin{array}{cccccc}
a_{1}+x & b_{2} & 0 & . & . & 0 \\
1 & a_{2}+x & b_{3} & . & . & 0 \\
0 & 1 & a_{3}+x & . & . & . \\
0 & 0 & 1 & . & . & 0 \\
. & . & & & 0 \\
. & . & & & . \\
. & . & . & . & a_{n-1}+x & b_{n} \\
0 & 0 & . & . & 1 & a_{n}+x
\end{array}\right| .
$$

This is seen directly when the determinant is expanded according to the elements of the last row or column.

If we denote this determinant by $D(a, b, 1, x)$, we may also write it in the equivalent form $D\left(a, b^{1 / 2}, b^{1 / 2}, x\right)$, which is formed by replacing the elements immediately above and immediately to the left of each principal diagonal element $a_{r, r}$ by $b_{r}{ }^{1 / 2}$, $r=2, \cdots, n$. This is a symmetric secular determinant with real elements, since the constants $b_{r}$ are positive by (7). From this follows again that the roots of $y_{n}$ are all real.*

* Kowalewski, Determinantentheorie, 1909, p. 126. 
6. Representation by Derivatives. The orthogonal polynomials $y_{n}$ are representable as derivatives of order $n$ by the formula*

$$
h_{n} y_{n}=(1 / \rho) D_{x}^{n}\left(\rho p^{n}\right),
$$

where $h_{n}$ is the coefficient of $x^{n}$ on the right.

If, in the expression

$$
L(y) \equiv p y_{n}^{\prime \prime}+q y_{n}^{\prime}+\lambda_{n} y_{n}
$$

where the coefficients are as in (4), we substitute $u_{n}=\rho y_{n}$, we obtain

$$
M(u) \equiv p u_{n}^{\prime \prime}+\left(2 p^{\prime}-q\right) u_{n}^{\prime}+\left(\lambda_{n}+p^{\prime \prime}-q^{\prime}\right) u_{n} .
$$

We now form the expression

where

$$
N(v) \equiv p v_{n}^{\prime \prime}-\left[(n-2) p^{\prime}+q\right] v_{n}^{\prime}+\mu_{n} v_{n},
$$

$$
\mu_{n}=\lambda_{n}+\frac{1}{2}(n-1)(n-2) p^{\prime \prime}+(n-1) q^{\prime}=1-2(n-1) \alpha .
$$

Differentiation of $N(v) n$ times gives $M(v)$. Also $v_{n}=\rho p^{n}$ gives

$$
N\left(\rho p^{n}\right)=\rho p^{n}\left[\mu_{n}+q^{\prime}+(n-1) p^{\prime \prime}\right]=0 .
$$

Therefore the expression on the right of (12) is a solution of $L(y)=0$. But this expression is readily shown to be a polynomial of degree $n$ by use of the relation $d(\rho p) / d x=\rho q$. Hence it differs from $y_{n}$ at most by a constant factor.

7. Generating Function. The orthogonal polynomials $y_{n}$ have a generating function

$$
\phi(x, t)=\frac{\rho(y)}{\rho(x)} \frac{\partial y}{\partial x}, \text { where } y=x+t p(y) .
$$

The Lagrange expansion formula gives the formal result

$$
\rho(y) \frac{\partial y}{\partial x}=\rho(x)+\rho(x) \sum_{1}^{\infty} D_{x}^{n}\left(\rho p^{n}\right) t^{n} / n ! .
$$

Division by $\rho(x)$ and taking account of (12) gives

$$
\phi(x, t)=\sum_{0}^{\infty} h_{n} y_{n} t^{n} / n !
$$

\footnotetext{
* See Abramescu, loc. cit., pp. 213-14, 217.
} 
ExAmpLE. We apply these results to the Laguerre polynomials considered by G. Szegö.* They are regarded as arising by the orthogonalization of

$$
x^{n+k} g(x) \equiv e^{-x / 2} x^{n+k} ; \quad k \geqq 0 ; \quad(a, b)=(0, \infty) .
$$

Then $p(x)=\beta x ;$ also, $d(\rho p) / d x=\rho q$ gives $q=(2 k+1-x) / \beta$. Taking $\beta=1$, we have the differential equation in our standard form

$$
x y_{n}^{\prime \prime}+(2 k+1-x) y_{n}^{\prime}+n y_{n}=0 .
$$

This gives $C_{1}^{(n)}$ and $C_{2}{ }^{(n)}$, then $a_{n}$ and $b_{n}$; hence the difference equation is

$$
y_{n}=(1-2 k-2 n+x) y_{n-1}-(n-1)(n+2 k-1) y_{n-2} .
$$

The normalizing constants are

$$
\begin{aligned}
1 / c_{n}^{2} & =\prod_{m=2}^{n+1}(m-1)(m+2 k-1) \int_{0}^{\infty} e^{-x} x^{2 k} d x \\
& =\prod_{m=2}^{n+1}(m-1)(m+2 k-1) \Gamma(2 k+1)
\end{aligned}
$$

The generating function is

$$
\frac{\rho(v)}{\rho(x)} \frac{\partial y}{\partial x}, \quad\left(y=x+t y, \rho(x)=e^{-x} x^{2 k}\right)
$$

which gives at once

$$
\phi(x, t)=(1-t)^{-2 k-1} e^{-x t /(1-t)} .
$$

8. The Case $Q(x) \equiv 0$ in (1). The considerations in $\$ 2$ show that we must have $\lambda_{1}=0$.

Then the differential equation must be

$$
p y_{n}^{\prime \prime}+\lambda_{n} y_{n}=0
$$

where

$$
\begin{aligned}
p=\alpha x^{2}+\beta x+\gamma & =-\alpha(x-a)(b-x), \alpha \neq 0 ; \\
\lambda_{n} & =-n(n-1) \alpha .
\end{aligned}
$$

Hence we may write

* Ein Beitrag zur Theorie der Polynome von Laguerre und Jacobi, Mathematische Zeitschrift, vol. 1 (1918). 


$$
(x-a)(b-x) y_{n}^{\prime \prime}+n(n-1) y_{n}=0 .
$$

Then $\rho=1 / p$, and $\int_{a}^{b} \rho d x$ does not exist. From $\left(3^{\prime \prime}\right), y_{n}$ vanishes at $a$ and $b, n>1$, and therefore

$$
\int_{a}^{b} \frac{1}{p} y_{n} y_{m} d x=\left(y_{n} y_{m}^{\prime}-y_{m} y_{n}^{\prime}\right)_{a}^{b}=0, \quad n \neq m ; n, m>1 .
$$

Hence the polynomials form an incompletely orthogonal system. The normalizing constants are given by

$$
1 / c_{n}^{2}=\int_{a}^{b}\left(y_{n}^{2} / p\right) d x, \quad n>1,
$$

and $c_{0}$ and $c_{1}$ do not exist. Other results of the preceding sections require corresponding restrictions.

9. Exceptional Cases. The exceptional case $\lambda_{n}-\lambda_{n-t}=0$; $n>1, t=2,3, \cdots, n$.

The value of $\lambda_{n}$ from (4) gives

$$
\lambda_{n}-\lambda_{n-t}=\alpha t(1 / \alpha+1+t-2 n)=0 .
$$

Therefore $1 / \alpha$ must be a positive integer. It follows that in general, solutions $y_{n}$ will not exist as polynomials for the following values of $n$ :

when $1 / \alpha=2 m, \quad$ for $n=m+1, m+2, \cdots, 2 m+1$, when $1 / \alpha=2 m-1$, for $n=m+1, m+2, \cdots, 2 m$,

$$
(m=1,2,3, \cdots) \text {. }
$$

(Exceptions consist of cases where individual constants $C_{t}{ }^{(n)}$ become indeterminate and may be given arbitrary values.) The differential equation furnishes an incomplete system of polynomials, which, since $\alpha>0$, will not be orthogonal.

For example, the equation

$$
\left(x^{2} / 4-1\right) y_{n}^{\prime \prime}-(x+1) y_{n}^{\prime}+[n(5-n) / 4] y_{n}=0
$$

has the solutions $y_{0}=1, y_{1}=x+1, y_{2}=x^{2}+4 x+4 ; y_{3}, y_{4}$, and $y_{5}$ do not exist as polynomials of degree $3,4,5$, respectively; then we again have polynomial solutions

$$
y_{6}=x^{6}-4 x^{5}-4 x^{4}+32 x^{3}-16 x^{2}-64 x+64,
$$

and so on.

The University of Nebraska 